\title{
Variations on Bochner-Riesz multipliers in the plane
}

\author{
by
}

Daniele Debertol (Pisa)

Abstract. We consider the multiplier $m_{\mu}$ defined for $\xi \in \mathbb{R}^{2}$ by

$$
m_{\mu}(\xi) \doteq\left(\frac{1-\xi_{1}^{2}-\xi_{2}^{2}}{1-\xi_{1}}\right)^{\mu} 1_{D}(\xi)
$$

$D$ denoting the open unit disk in $\mathbb{R}^{2}$. Given $\left.p \in\right] 1, \infty[$, we show that the optimal range of $\mu$ 's for which $m_{\mu}$ is a Fourier multiplier on $L^{p}$ is the same as for Bochner-Riesz means. The key ingredient is a lemma about some modifications of Bochner-Riesz means inside convex regions with smooth boundary and non-vanishing curvature, providing a more flexible version of a result by Iosevich et al. [Publ. Mat. 46 (2002)]. As an application, we show that the same characterization also holds true for the multiplier $p_{\mu}(\xi) \doteq\left(\xi_{2}-\xi_{1}^{2}\right)_{+}^{\mu} \xi_{2}^{-\mu}$. Finally, we briefly discuss the $n$-dimensional analogue of these results.

1. Introduction. Let us consider the two-parameter family of functions given by

$$
m_{(\nu, \mu)}(\xi) \doteq\left(1-\xi_{1}\right)^{\nu-\mu}\left(1-|\xi|^{2}\right)_{+}^{\mu}
$$

For a fixed $\nu \neq 0$, a complete characterization of the range of $\mu$ 's such that $m_{(\nu, \mu)}$ belongs to $M_{p}$, the space of Fourier multipliers on $L^{p}\left(\mathbb{R}^{2}\right)$, was given in [5]. In particular, for strictly positive $\nu$ and $2<p<\infty$, it was shown there that

$$
m_{(\nu, \mu)} \in M_{p} \Leftrightarrow \mu>\max \{1 / 2-2 / p, 0\} .
$$

In this paper we remove the restriction on $\nu$. That is, since for $\nu<0$ the function $m_{(\nu, \mu)}$ is clearly unbounded, we prove that (1.1) continues to hold when $\nu=0$. Therefore, we shall only have to deal with $m_{(0, \mu)}$ and we change the notation accordingly, letting $m_{\mu} \doteq m_{(0, \mu)}$,

$$
m_{\mu}(\xi)=\left(1-\xi_{1}\right)^{-\mu}\left(1-|\xi|^{2}\right)_{+}^{\mu} .
$$

First of all, note that $m_{\mu}$ has a discontinuity at $(1,0)$, so that it cannot be an $L^{1}$ multiplier for any choice of $\mu$. Therefore, with this (trivial) exception, (1.1) shows in fact that the more singular $m_{\mu}$ exhibits the same $(p, \mu)$ re-

2000 Mathematics Subject Classification: Primary 42B15.

Key words and phrases: Bochner-Riesz multipliers, homogeneous multipliers. 
gion of boundedness of the classical Bochner-Riesz means on the disk (see e.g. [3]).

Further, $m_{\mu}$ can actually be thought of as a model case of Bochner-Riesz means with an additional singularity, for the factor $\left(1-\xi_{1}\right)^{\delta} \phi\left(\xi_{1}\right)$, with $\delta$ strictly positive and $\phi$ any compactly supported and sufficiently smooth function, is an $L^{1}$ multiplier.

In particular, this means that the case $\nu=0$ entails the one with $\nu>0$, up to $L^{1}$ results.

Thus, necessary conditions essentially being the same as the ones in [5], we shall mainly concentrate on the "if" implication of (1.1). The approach we use here relies on the idea that there is some kind of non-isotropic homogeneity underlying $m_{\mu}$, best reflected by the behaviour of the closely related multiplier $p_{\mu}(\xi) \doteq\left(\xi_{2}-\xi_{1}^{2}\right)_{+}^{\mu} \xi_{2}^{-\mu}$. These considerations have already been taken up in [5], where some evidence supported by [2] also suggested it was quite natural to conjecture that at least (1.1) is the correct answer for the "model case" $p_{\mu}$.

In point of fact, the strategy we adopt is the following: first, we apply the slicing lemma of [8], which clearly has a flavour of homogeneity (but see also the formulation given in [1]), to $m_{\mu}$. Since $m_{\mu}$ has sufficient Lipschitz regularity, this has the effect of reducing the problem to the uniform estimate of a multiplier norm for these slices, whose main point is to make the worst singularity at $(1,0)$ become harmless. After some preparation, Lemma 1.1 below will finally allow us to conclude.

LeMma 1.1. Let $E$ be a convex symmetric body with non-empty interior and non-vanishing curvature, and $g$ a smooth, first-order defining function for its boundary. Quantitatively, assume that

(1) the curvature $k$ of $\partial E$ is bounded below away from zero, $k \geq A_{1}>0$;

(2) $g \in C_{\mathrm{c}}^{\infty}\left(\mathbb{R}^{2}\right)$ is a concave function in a neighbourhood of $E$ such that

$$
\partial E=E \cap\{g \equiv 0\} \quad \text { and } \quad|\nabla g| \geq A_{2}>0 \text { on } \partial E ;
$$

(3) the spectral norm of the Hessian of $g$ is bounded on $E$,

$$
\max \{|\lambda|: \lambda \text { an eigenvalue of } H g\} \leq A_{3} .
$$

Further, let $\mu>0$ and $4 \leq p \leq \infty$. Then $\left\|g^{\mu} 1_{E}\right\|_{M_{p}}$ is finite if and only if $\mu>1 / 2-2 / p$, and in this case it only depends on the $A_{j}$ 's, on a Schwartz seminorm of $g$ and on $\mu$.

We remark that the core of Lemma 1.1 is the deep result $[7,1.1]$, which however does not apply directly in our situation. The version provided here extends both $[7,1.1]$ and $[9$, Thm. 1$]$ under the additional assumption of nonvanishing curvature of $E$. Moreover, in order to prove uniform estimates, we need an explicit control over the geometric constants that occur in the proof. 
Note also that an $n$-dimensional analogue is available for Lemma 1.1, whose conclusion is once more deduced from $[7,1.1]$.

In the last part of the paper, we give an application of Theorem 2.1 to the case of $p_{\mu}$, showing that (1.1) was indeed the right guess, and we look for a partial generalization of the above pattern to the $n$-dimensional setting of the same problem.

Finally, I want to express my deep gratitude to Tony Carbery and Fulvio Ricci for the stimulating conversations they shared with me on these subjects.

2. Main result. Our goal is to prove the next theorem. When $\mu=0$, note that of course Fefferman's result [6] applies.

THEOREM 2.1. Let $1 \leq p \leq \infty, \mu>0$. Then $m_{\mu}$ is an $L^{p}\left(\mathbb{R}^{2}\right)$ multiplier if and only if $p \in] 1, \infty[$ and $\mu>\max \{|2 / p-1|-1 / 2,0\}$.

Proof. According to the previous remarks, the operator with multiplier $m_{\mu}$ can never be bounded if $p=1$, for any $\mu$. The remaining necessary conditions have to hold because of $[5,2.1]$, so that by duality and interpolation we are only left with showing the positive part of (1.1) when $p \geq 4$. To this end, we need to quote a lemma which is a special case of Corollary 2.ii in [8]. But first, we have to introduce some notation.

Let us define a family of anisotropic dilations by

$$
t \cdot\left(\xi_{1}, \xi_{2}\right) \doteq\left(t \xi_{1}, t^{2} \xi_{2}\right), \quad t>0,
$$

and let $\phi$ be any $C^{\infty}$ bump function whose support is a shell adapted to these dilations.

Also, denote by $\Lambda_{\alpha}$ the usual non-homogeneous Lipschitz space of order $\alpha>0$, and for a function $m$ finally let

$$
m_{(t)}(\xi) \doteq m(t \cdot \xi) .
$$

Lemma 2.2 ([8, Cor. 2]). Assume that $m$ satisfies

(a) $\sup _{t>0}\left\|\phi m_{(t)}\right\|_{M_{q}}<\infty$ for some finite $q>2$,

(b) $\sup _{t>0}\left\|\phi m_{(t)}\right\|_{\Lambda_{\alpha}}<\infty$ for some $\alpha>0$.

Then $m \in M_{p}$ for those $p$ such that $|1 / p-1 / 2|<1 / 2-1 / q$.

Actually, in order to apply Lemma 2.2, it is more convenient to compose $m_{\mu}$ with an affine transformation moving its singularity to the origin. Since multiplier norms are invariant under such changes of coordinates, the conclusion of Theorem 2.1 is unaffected.

Therefore, we consider the function

$$
g_{\mu}(\xi) \doteq\left(2 \xi_{2}-\xi_{2}^{2}-\xi_{1}^{2}\right)_{+}^{\mu} \xi_{2}^{-\mu},
$$


which also exhibits a nicer behaviour under the selected family of dilations. Indeed, note that

$$
g_{\mu}(t \cdot \xi)=\left(2 \xi_{2}-t^{2} \xi_{2}^{2}-\xi_{1}^{2}\right)_{+}^{\mu} \xi_{2}^{-\mu} .
$$

In particular, $\phi g_{\mu}(t \cdot) \not \equiv 0$ only for small $t$.

Now, let us start by showing that $g_{\mu}$ satisfies part (b) of the hypothesis of the lemma. A first trivial observation is that, since we may assume that $t$ is bounded above, there exist $C^{\infty}$ functions $\chi$ and $\vartheta$, compactly supported in the open upper half-plane and on the real line respectively, such that

$$
\phi(\xi) g_{\mu}(t \cdot \xi)=\left(\left(\vartheta(y) y_{+}^{\mu}\right) \circ\left(\left(2 \xi_{2}-t^{2} \xi_{2}^{2}-\xi_{1}^{2}\right) \chi(\xi)\right)\right) \phi(\xi) \chi(\xi) \xi_{2}^{-\mu} .
$$

Further, it is easy to see that:

(i) $\|f g\|_{\Lambda_{\alpha}} \leq\|f\|_{\Lambda_{\alpha}}\|g\|_{\Lambda_{\alpha}}$;

(ii) $\|f \circ g\|_{\Lambda_{\alpha}} \leq\|f\|_{\Lambda_{\alpha}}\left(1+\|g\|_{C^{1}}^{\alpha}\right), 0<\alpha<1$.

In particular, (i) allows us to assume $\mu<1$.

Moreover, the $C^{1}$ norms of $\chi(\xi)\left(2 \xi_{2}-t^{2} \xi_{2}^{2}-\xi_{1}^{2}\right)$ are uniformly bounded for $t$ small, and the factor $\phi(\xi) \chi(\xi) \xi_{2}^{-\mu}$ is smooth and compactly supported.

Since $\vartheta(y) y_{+}^{\mu}$ belongs to $\Lambda_{\mu+1 / 2}^{2, \infty}(\mathbb{R})$, by $(4.12)$ in $[5]$, and $\Lambda_{\mu}(\mathbb{R})$ contains the latter space (see e.g. [10]), the claim follows by applying (i) and (ii) to $(2.1)$.

Therefore, it only remains to show that $g_{\mu}$ satisfies part (a) of the lemma. The condition in (1.1) being defined by a strict inequality, we may let $q \doteq p$.

As before, we can get rid of the singular factor $\xi_{2}^{-\mu}$, which in fact is no longer so once it is coupled with $\chi$, for it boils down to a Schwartz function. In the end, we are done if we can prove the uniform estimate

$$
\sup _{t \leq C}\left\|\phi(\xi) \chi(\xi)\left(2 \xi_{2}-t^{2} \xi_{2}^{2}-\xi_{1}^{2}\right)_{+}^{\mu}\right\|_{M_{p}}<\infty .
$$

In view of Lemma 1.1, still to be proven, we can assume that $C$ is as close to zero as we wish. In this case, we can further decompose $\chi$ as $\chi_{1}+\chi_{2}+\chi_{3}$, in such a way that $\chi_{1}+\chi_{3}$ is an even function of $\xi_{1}, \chi_{1}$ is supported in a right half-plane, and the factor $2 \xi_{2}-t^{2} \xi_{2}^{2}-\xi_{1}^{2}$ is bounded below away from zero independently of $t$ on the support of $\chi_{2}$. Consequently,

$$
\begin{aligned}
& \left\|\phi(\xi) \chi(\xi)\left(2 \xi_{2}-t^{2} \xi_{2}^{2}-\xi_{1}^{2}\right)_{+}^{\mu}\right\|_{M_{p}} \\
& \quad \leq\|\phi\|_{M_{1}}\left(2\left\|\chi_{1}(\xi)\left(2 \xi_{2}-t^{2} \xi_{2}^{2}-\xi_{1}^{2}\right)_{+}^{\mu}\right\|_{M_{p}}+\left\|\chi_{2}(\xi)\left(2 \xi_{2}-t^{2} \xi_{2}^{2}-\xi_{1}^{2}\right)^{\mu}\right\|_{M_{1}}\right),
\end{aligned}
$$

and the second summand is bounded uniformly in $t$, if $t \ll 1$, for so are the Schwartz seminorms of every order of $\chi_{2}(\xi)\left(2 \xi_{2}-t^{2} \xi_{2}^{2}-\xi_{1}^{2}\right)^{\mu}$, by construction.

Therefore, (2.2) is established as soon as we prove

$$
\sup _{t \ll 1}\left\|\chi_{1}(\xi)\left(2 \xi_{2}-t^{2} \xi_{2}^{2}-\xi_{1}^{2}\right)_{+}^{\mu}\right\|_{M_{p}}<\infty .
$$


Note that (2.3) closely resembles the kind of Bochner-Riesz means studied in [9], for instance. Actually, the next step consists in replacing the "global" factor $2 \xi_{2}-t^{2} \xi_{2}^{2}-\xi_{1}^{2}$ in $(2.3)$ with a better localized one. More precisely, we aim at producing functions $\eta_{t} \in C_{\mathrm{c}}^{\infty}\left(\mathbb{R}^{2}\right)$ for which

$$
\chi_{1}(\xi)\left(2 \xi_{2}-t^{2} \xi_{2}^{2}-\xi_{1}^{2}\right)=\chi_{1}(\xi) \eta_{t}(\xi),
$$

with the additional property that, after a suitable affine transformation, the bounded connected component $E_{t}$ of $\left\{\eta_{t} \geq 0\right\}$ satisfies the hypotheses of Lemma 1.1 uniformly in $t$.

Apart from the technicalities, what we do simply consists in replacing the portions of the ellipses $\left\{2 \xi_{2}-t^{2} \xi_{2}^{2}-\xi_{1}^{2} \equiv 0\right\}$ inside supp $\chi_{1}$ with a family of convex sets whose curvature and diameter are better behaved, and in particular, uniformly bounded for $t$ small.

That this is indeed possible can be shown as follows. Let first $\psi, \beta$ be two smooth functions on $\mathbb{R}$, respectively supported in $[-a, \infty[$ and $[-2 b, 2 b]$ for some strictly positive $a, b$, which will only depend on $\chi_{1}$, such that $\{\psi, \psi(-\cdot)\}$ is a partition of unity and $\beta \equiv 1$ on $[-b, b]$. In addition, take a rigid motion $R_{t}$ of the plane for which $R_{t}\left(\left\{2 \xi_{2}-t^{2} \xi_{2}^{2}-\xi_{1}^{2} \equiv 0\right\} \cap \operatorname{supp} \chi_{1}\right)$ may be expressed as the graph $(\gamma(s), s)$ of a smooth function $\gamma$ with $\gamma(0)=2 a$, $\gamma^{\prime}(0)=0$. Then, if $J$ denotes the reflection with respect to the origin, $J \xi \doteq-\xi$, we can let

$$
\begin{aligned}
\eta_{t}\left(R_{t}^{-1} \xi\right) \doteq & \psi\left(\xi_{1}\right) \beta\left(\xi_{2}\right)\left(2 \xi_{2}-t^{2} \xi_{2}^{2}-\xi_{1}^{2}\right)\left(R_{t}^{-1} \xi\right) \\
& +\psi\left(-\xi_{1}\right) \beta\left(\xi_{2}\right)\left(2 \xi_{2}-t^{2} \xi_{2}^{2}-\xi_{1}^{2}\right)\left(R_{t}^{-1} J \xi\right)
\end{aligned}
$$

Since the hypotheses of Lemma 1.1 are invariant under rotations and translations, and the Hessian of $\eta_{t}$ is negative definite where $\eta_{t}$ is strictly positive, the claim is established.

Thus, by (2.4) we obtain

$$
\left\|\chi_{1}(\xi)\left(2 \xi_{2}-t^{2} \xi_{2}^{2}-\xi_{1}^{2}\right)_{+}^{\mu}\right\|_{M_{p}} \leq\left\|\chi_{1}\right\|_{M_{1}}\left\|\eta_{t}^{\mu} 1_{E_{t}}\right\|_{M_{p}}
$$

so that (2.3) and hence Theorem 2.1 are finally established once we prove Lemma 1.1, which we do now:

Proof of Lemma 1.1. Let $h$ denote the symmetric part of $g, h(\xi) \doteq$ $(g(\xi)+g(-\xi)) / 2$. By the mean value theorem, the absolute value of either $\partial_{1} g$ or $\partial_{2} g$ is bounded below by $A_{2} / 2$ on each ball of radius smaller than $A_{2} /\left(8 A_{3}\right)$ centred at $\xi \in \partial E$. In particular, we find

$$
\left|\partial_{i} g(\xi)\right|+\left|\partial_{i} g(-\xi)\right|=\left|\partial_{i} h(\xi)-\partial_{i} h(-\xi)\right| \leq 2 A_{3}|\xi|
$$

$i=1,2$, and therefore the minimum distance from $\partial E$ to the origin is bounded below by $A_{2} /\left(4 A_{3}\right)$. Note also that the diameter of $E$ is not greater than $4 / A_{1}$, by convexity. Further, for $\xi \in \partial E$ we have 


$$
g(0)=h(0)=\int_{-1}^{0} \xi \cdot \nabla h(s \xi) d s
$$

and hence, by the arbitrariness of $\xi$ we obtain

$$
2 g(0) \geq|\xi||\nabla h(-\xi)||\{s \in[-1,0]: \xi \cdot \nabla h(s \xi) \geq \xi \cdot \nabla h(-\xi) / 2\}|,
$$

and we may conclude that $g(0)$ too is bounded below in terms of $A_{2}$ and $A_{3}$. Thus, by concavity of $g$ and a Taylor expansion with integral remainder we have

$$
\xi \cdot \nabla g(-\xi)=g(0)-\int_{0}^{1} \int_{0}^{1} s^{t} \xi H g(-s u \xi) \xi d u d s \geq g(0)
$$

for $\xi$ on $\partial E$.

Now, let $\varrho$ denote the Minkowski functional for $E$, that is, $\varrho$ is homogeneous of degree 1 with respect to isotropic dilations and $\varrho \equiv 1$ on $\partial E$. By Dini's theorem, $\varrho$ is smooth away from the origin, and

$$
\nabla \varrho(\xi)=\nabla g(\xi) /(\xi \cdot \nabla g(\xi))
$$

on $\partial E$. Moreover, (2.5) implies that Schwartz seminorms of $\varrho$ over compact subsets of $E \backslash\{0\}$ are controlled by Schwartz seminorms of $g$ and by the $A_{j}$ 's. Finally, letting $\xi_{0} \doteq \xi / \varrho(\xi)$, we arrive at

$$
|1-\varrho(\xi)|=\left|\xi-\xi_{0}\right| /\left|\xi_{0}\right|<1 / 2
$$

for $\xi$ in $U \doteq\left\{\xi: \mathrm{d}(\xi, \partial E)<A_{2} /\left(8 A_{3}\right)\right\}$.

Now, since $E$ is bounded, we may take a partition of unity $\beta_{1}, \ldots, \beta_{N}$ of the circle in such a way that each $\beta_{i}$ is obtained by composing $\beta_{1}$ with a rotation and that their number $N$ is bounded above by the $A_{j}$ 's. Let

$$
\widetilde{\beta}_{i}(\xi) \doteq \beta_{i}(\xi /|\xi|), \quad \xi \neq 0
$$

then, for any $i$, at least one of $\psi_{1}(\xi) \doteq\left(\xi_{1}, \varrho(\xi)\right)$ and $\psi_{2}(\xi) \doteq\left(\varrho(\xi), \xi_{2}\right)$ is a coordinate patch on the support of $\widetilde{\beta}_{i}$, and in each case the corresponding jacobian is bounded above and below by the $A_{j}$ 's and $\|g\|_{C^{1}}$. Let us work, for instance, with $\psi_{1}$ (the case of $\psi_{2}$ may be treated similarly), and define $\widetilde{g} \doteq g \circ \psi_{1}^{-1}$. If we freeze $\xi_{1}$, then a second-order Taylor expansion of $\widetilde{g}\left(\xi_{1}, \cdot\right)$ centred at $\varrho=1$ gives us

$$
\widetilde{g}\left(\xi_{1}, \varrho\right)=(1-\varrho)\left(-\partial_{\varrho} \widetilde{g}\left(\xi_{1}, 1\right)+(1-\varrho) \int_{0}^{1} \int_{0}^{1} s \partial_{\varrho \varrho} \widetilde{g}\left(\xi_{1}, 1-s u(1-\varrho)\right) d u d s\right),
$$

and going back to the $\xi=\left(\xi_{1}, \xi_{2}\right)$ variables yields

$$
\begin{aligned}
g(\xi)= & (1-\varrho(\xi))\left(-\psi_{1}^{-1}\left(\xi_{1}, 1\right) \cdot \nabla g\left(\psi_{1}^{-1}\left(\xi_{1}, 1\right)\right)\right. \\
& \left.+(1-\varrho(\xi)) \iint_{0}^{1} s \partial_{\varrho \varrho} \widetilde{g}\left(\xi_{1}, 1-s u(1-\varrho(\xi))\right) d u d s\right) \\
= & (1-\varrho(\xi)) g_{i}(\xi) .
\end{aligned}
$$


By (2.5), we can therefore select $\varepsilon>0$ so small, only depending on $A_{j}$ 's and on a suitable Schwartz seminorm of $g$, that $\partial E+B(0, \varepsilon) \subset U$ and that the factor $g_{i}$ in $(2.7)$ be a strictly positive (and smooth) function bounded below by the same quantities.

Now we are almost done: take a bump function $\varphi_{0} \in C_{\mathrm{c}}^{\infty}(\mathbb{R})$ whose support is contained in $[1-\varepsilon, 1+\varepsilon]$, and let $\varphi \doteq \varphi_{0} \circ \varrho$. Then $(1-\varphi) g^{\mu} 1_{E}$ is a Schwartz function whose $M_{1}$ norm merely depends on $\varepsilon$ and on the other desired constants, and we are left with

$$
\varphi g^{\mu} 1_{E}=\sum_{i=1}^{N} \varphi(1-\varrho)_{+}^{\mu} g_{i}^{\mu} \widetilde{\beta}_{i} .
$$

By this decomposition, it is finally easy to conclude that $g^{\mu} 1_{E}$ is an $M_{p}$ multiplier if and only if $(1-\varrho)_{+}^{\mu}$ is, and therefore we can apply $[7,1.1]$. Moreover, by examining their proof carefully, one can see that in the case of non-vanishing curvature the multiplier norm of $(1-\varrho)_{+}^{\mu}$ depends exclusively on the $A_{j}$ 's.

As a consequence of (2.3), the proof of Theorem 2.1 is also complete.

Note that when $\mu=0$, the proof of [6] can effortlessly be adapted in order to obtain the following version of Lemma 1.1:

REMARK 2.3. If $E$ is a convex body with $C^{2}$ boundary and non-vanishing curvature, then $1_{E}$ belongs to $M_{p}$ if and only if $p=2$.

REMARK 2.4. The same characterizations of Theorem 2.1 and Remark 2.3 also hold for the multiplier $p_{\mu}$ introduced before, according to whether $\mu$ is strictly positive or $\mu=0$.

Indeed, positive results can be deduced by Theorem 2.1 via the same argument used in [5]; for negative ones, $p \neq 1$ comes by discontinuity of the multiplier, as before, and otherwise we can apply Remark 2.3 and Lemma 1.1 to a convex symmetric body sharing with the parabola a piece of boundary away from the origin.

Finally, a few words about a possible way to generalize these results to an $n$-dimensional setting.

In fact, thinking of the way multipliers of the form $m_{\mu}$ first arose in $[4,5]$, it seems natural to ask the same questions for the two families of multipliers on $\mathbb{R}^{n}$,

$$
m_{\mu}^{(n)}(\xi) \doteq\left(1-\xi_{1}\right)^{-\mu}\left(1-\xi_{1}^{2}-\cdots-\xi_{n}^{2}\right)_{+}^{\mu}
$$

and

$$
p_{\mu}^{(n)}(\xi) \doteq\left(\xi_{n}-\xi_{1}^{2}-\cdots-\xi_{n-1}^{2}\right)_{+}^{\mu} \xi_{n}^{-\mu} .
$$

Of course, what we obtain for $n \geq 3$ is far from the full strength of the 2-dimensional characterization: 
REMARK 2.5. Let $n \geq 3, p>2$. Then a necessary condition for $m_{\mu}^{(n)}$ to be a Fourier multiplier on $L^{p}\left(\mathbb{R}^{n}\right)$ is that

$$
\mu>\max \{n(1 / 2-1 / p)-1 / 2,0\} .
$$

If $p \geq 4$, condition (2.8) is also sufficient. In both cases, the same conclusion holds for $p_{\mu}^{(n)}$.

As a matter of fact, most of the proof of Theorem 2.1 can actually go unchanged towards obtaining Remark 2.5.

Indeed, both Lemma 2.2 and Lemma 1.1 are $n$-dimensional by nature (see again [8, Cor. 2] and [7, 1.1], respectively). However, the conclusion of Lemma 1.1 which we may draw from Theorem 1.1 in [7] is now much weaker than before: this is so because condition (2.8), which is necessary for every $p>2$, is still only sufficient for $p \geq 4$, independently of $n$.

Since the critical exponent for $[7,1.1]$ is $p=2 n /(n-1)$, which is strictly smaller than 4 as soon as $n>2$, a corresponding gap is left open in Remark 2.5.

\section{References}

[1] A. Carbery, Variants of the Calderón-Zygmund theory for $L^{p}$-spaces, Rev. Mat. Iberoamericana 2 (1986), 381-396.

[2] A. Carbery and A. Seeger, Homogeneous Fourier multipliers of Marcinkiewicz type, Ark. Mat. 33 (1995), 45-80.

[3] A. Córdoba, A note on Bochner-Riesz operators, Duke Math. J. 46 (1979), 505-511.

[4] D. Debertol, Besov spaces and the boundedness of weighted Bergman projections over symmetric tube domains, Publ. Mat. 49 (2005), 21-72.

[5] -, A family of Bochner-Riesz type multipliers on the two-dimensional disk, J. Math. Anal. Appl. 317 (2006), 192-206.

[6] C. Fefferman, The multiplier problem for the ball, Ann. of Math. 94 (1971), 330-336.

[7] A. Iosevich, E. Sawyer and A. Seeger, Two problems associated with convex finite type domains, Publ. Mat. 46 (2002), 153-177.

[8] A. Seeger, Some inequalities for singular convolution operators in $L^{p}$-spaces, Trans. Amer. Math. Soc. 308 (1988), 259-272.

[9] P. Sjölin, Fourier multipliers and estimates of the Fourier transform of measures carried by smooth curves in $\mathbb{R}^{2}$, Studia Math. 51 (1974), 169-182.

[10] E. M. Stein, Singular Integrals and Differentiability Properties of Functions, Princeton Univ. Press, Princeton, 1970.

Scuola Normale Superiore

Piazza dei Cavalieri 7

56126 Pisa (PI), Italy

E-mail: debertol@sns.it 